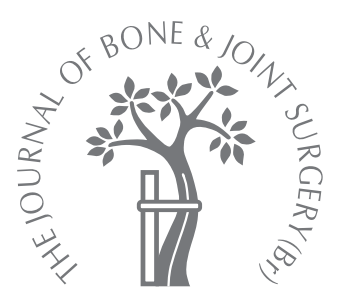

T. Takebayashi, J. M. Cavanaugh, S. Kallakuri, C. Chen, T. Yamashita

From Sapporo Medical University School of Medicine, Sapporo, Japan
- T. Takebayashi, MD, PhD, Orthopaedic Surgeon - T. Yamashita, MD, PhD, Professor and Chairman Department of Orthopaedic Surgery

Sapporo Medical University School of Medicine, South-1, West-16, Chuo-ku, Sapporo 060-8543, Japan.

II J. M. Cavanaugh, MD, PhD, Professor

S. Kallakuri, MS, Assistant Professor

C. Chen, MD, Assistant

Professor

Bioengineering Center

Wayne State University, 818 W.

Hancock, Detroit, Michigan

48201, USA.

Correspondence should be sent to $\mathrm{Dr}$ T. Takebayashi; e-mail: takebaya@sapmed.ac.jp

C2006 British Editorial Society of Bone and Joint Surgery doi:10.1302/0301-620X.88B4. $17194 \$ 2.00$

$J$ Bone Joint Surg [Br] 2006;88-B:554-7.

Received 15 September 2005;

Accepted after revision

15 December 2005

\title{
Sympathetic afferent units from lumbar intervertebral discs
}

To clarify the pathomechanisms of discogenic low back pain, the sympathetic afferent discharge originating from the L5-L6 disc via the L2 root were investigated neurophysiologically in $\mathbf{3 1}$ Lewis rats. Sympathetic afferent units were recorded from the L2 root connected to the lumbar sympathetic trunk by rami communicantes. The L5-L6 discs were mechanically probed, stimulated electrically to evoke action potentials and, finally, treated with chemicals to produce an inflammatory reaction. We could not obtain a response from any units in the L5-L6 discs using mechanical stimulation, but with electrical stimulation we identified 42 units consisting mostly of A-delta fibres. In some experiments a response to mechanical probing of the L5-L6 disc was recognised after producing an inflammatory reaction. This study suggests that mechanical stimulation of the lumbar discs may not always produce pain, whereas inflammatory changes may cause the disc to become sensitive to mechanical stimuli, resulting in nociceptive information being transmitted as discogenic low back pain to the spinal cord through the lumbar sympathetic trunk. This may partly explain the variation in human symptoms of degenerate discs.

Lumbar intervertebral discs are recognised as one of the major sources of low back pain. Elucidating the sensory innervation of the lumbar intervertebral disc and its adjacent tissues is important in understanding the mechanism of discogenic low back pain. It is accepted that lumbar intervertebral discs are innervated segmentally, with sinuvertebral nerves branching from the nerve fibres of the lumbar spine and the rami communicantes of the corresponding levels. ${ }^{1-3}$ Recently, it has been demonstrated in rats that the sinuvertebral nerves proceed into the paravertebral sympathetic trunk through the rami communicantes. ${ }^{2,4}$ Several immunohistological studies in the $\mathrm{rat}^{5-7}$ using retrograde neurotracer methods have shown innervation of the L5-L6 intervertebral discs and adjacent tissues from neurons of the L1 and L2 dorsal root ganglia, through the paravertebral sympathetic trunk.

Similarly, it has been reported in neurophysiological studies that mechanosensitive afferent units with receptive fields in the retroperitoneal space, including ventral aspects of the lumbar intervertebral disc and psoas muscles, can run through the paravertebral sympathetic trunk. ${ }^{8,9}$ In addition, these units responded to mechanical and chemical stimuli such as potassium chloride, hypertonic saline solution and bradykinin, which may indicate the involvement of nociceptive units. In the gastrointestinal system, it has been demonstrated that sympathetic C-fibre afferents with low and high thresholds can encode nociceptive information, such as excessive distension and ischaemia. ${ }^{10}$ Collectively, these studies imply that sensory information, including nociceptive stimulation from the lower lumbar intervertebral discs innervated by the sinuvertebral nerves, may be conducted by the paravertebral sympathetic trunk, via the rami communicantes, and then through the L1 and L2 dorsal root ganglia into the spinal cord. Although the sensory pathway from the lower lumbar intervertebral discs has been investigated both histologically and anatomically, the mechanisms of discogenic low back pain are still unknown.

Using neurophysiological techniques, we investigated in a rat model whether sympathetic afferent discharges originating from lower lumbar intervertebral discs and adjacent tissues pass through L2 dorsal roots as postulated and examined the properties of the afferent units.

\section{Materials and Methods}

All the surgical procedures were reviewed and approved by the Animal Investigation Committee at the institution where the experiments were carried out. The study was performed on 


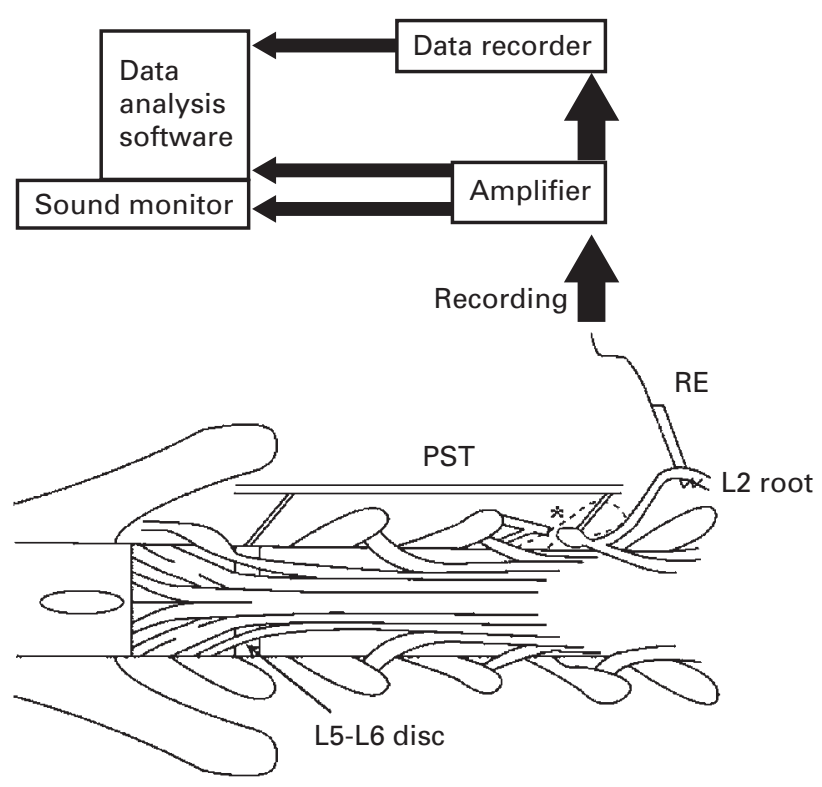

Fig. 1

Experimental design of the recording of sympathetic afferent units. Afferent impulses from L2 dorsal rootlets were amplified and recorded on an FM tape recorder. L2 spinal nerve roots connected only with rami communicantes to the lumbar paravertebral sympathetic trunk. $R E$, recording electrode; PST, paravertebral sympathetic trunk; cutting of the dorsal and ventral rami of $L 2$.

31 adult male Lewis rats weighing 350 to $400 \mathrm{~g}$. They were sedated and anaesthetised by an intramuscular injection of ketamine hydrochloride $(43 \mathrm{mg} / \mathrm{kg})$, xylazine $(7 \mathrm{mg} / \mathrm{kg})$ and torbutrol $(0.1 \mathrm{mg} / \mathrm{kg})$. Supplementary half doses were used as required to maintain skeletal muscle areflexia to toe pinch throughout the experiment.

A midline dorsal longitudinal incision was made over the lumbar spine and the multifidus muscles were removed along the spinous processes from T13 to S1. Only on the left-side, the paravertebral muscles were detached from the lumbar vertebral bodies. The dorsal ramus of the left spinal nerve of L2 was then carefully divided and the psoas muscles were exposed. The left transverse processes of L3 and L4 were partially resected and the ventral ramus of the left spinal nerve of L2, exposed beneath the vertebral body of L3, was divided distally to the dorsal root ganglion of L2. At this point, activity from peripheral receptive fields from the dorsal and ventral rami of L2 was abolished by dividing the rami so that the spinal nerve roots connected only with rami communicantes to the sympathetic chain. This was advantageous in preventing neural activity, except for that passing through the sympathetic chain and for minimising the influence of spinal movement caused by mechanical probing.

A laminectomy from L1 to L6 was performed with fine bone rongeurs. To prevent movement of the lumbar spine, especially at the L5-L6 intervertebral disc, the animal was tightly clamped at the 13th thoracic spinous process and the sixth lumbar transverse process. The dorsal nerve root of L2 was exposed. After dividing the dura to prevent desiccation, a pool was formed from skin flaps to hold warm $\left(37^{\circ} \mathrm{C}\right)$ mineral oil, in which the spinal cord and nerve roots were immersed.

Electrical extracellular recording. The left dorsal root of L2, detached from the spinal cord, was draped over a dualbipolar platinum recording electrode to examine the afferent units and was further divided to record from a smaller number of units (Fig. 1). The impulses on the two channels were amplified, monitored on an oscilloscope and an audiomonitor, digitised and analysed using computer-based spike discrimination and frequency analysis software. All software was part of the Computerscope Enhanced Graphics Acquisition and Analysis (EGAA) system (R. C. Electronics, Goleta, California). To allow later detailed analysis, we also recorded the data on an analogue tape recorder (MR30; TEAC, Montebello, California).

Mechanical stimulation. Lumbar structural elements, including the dorsal aspects of the L5-L6 intervertebral discs and adjacent facets and the dorsal side of the psoas muscles from L3 to L6, were mechanically probed with blunt glass rods and a 25 gauge needle. Once the receptive fields were identified, the mechanical threshold was measured using calibrated nylon filaments with a compression magnitude ranging from 0.4 to $48.3 \mathrm{~g}$ (Aesthesiometer; Stoelting, Wood Dale, Illinois). The test probing was performed separately on the bony lamina to ensure that these units were not also responsive to motion of the lumbar spine.

Electrical stimulation. If the disc and adjacent tissues did not respond to any mechanical stimulation, electrical stimulation with a bipolar electrical stimulator (1 to $20 \mathrm{~V}$ ) was applied to the dorsal aspects of the L5-L6 discs and adjacent tissues, to evoke action potentials and obtain latency. Later, based on the latencies and the distance between the recording electrode and the point of electrical stimulation, conduction velocity was estimated. The mechanically receptive areas were also electrically stimulated to allow calculation of the conduction velocity.

Chemical stimulation. Finally, $2 \%$ carrageenan $(0.1 \mathrm{ml})$ was applied to the L5-L6 disc in 19 of the 31 rats, to induce inflammation. The change in the discharge rate and the response of the L5-L6 disc to mechanical probing were observed and recorded.

Once the neurophysiological study was complete, the rats were killed with an intraperitoneal overdose of sodium pentobarbital and death assured by the production of bilateral pneumothoraces.

\section{Results}

Responses to mechanical stimuli. For mechanical stimulation, muscle units of the psoas were observed in 12 of the 31 experiments. No systematic attempt was made to evaluate the mechanical thresholds quantitatively, because of the 


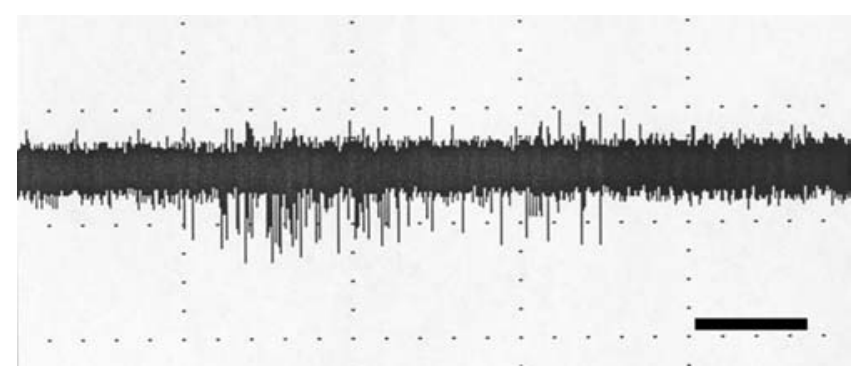

Fig. 2

Response of dorsal root ganglia units to mechanical stimulation. The discharge pattern was sporadic and did not seem to be directly influenced by mechanical stimulation. Black bar indicates $1 \mathrm{~s}$.

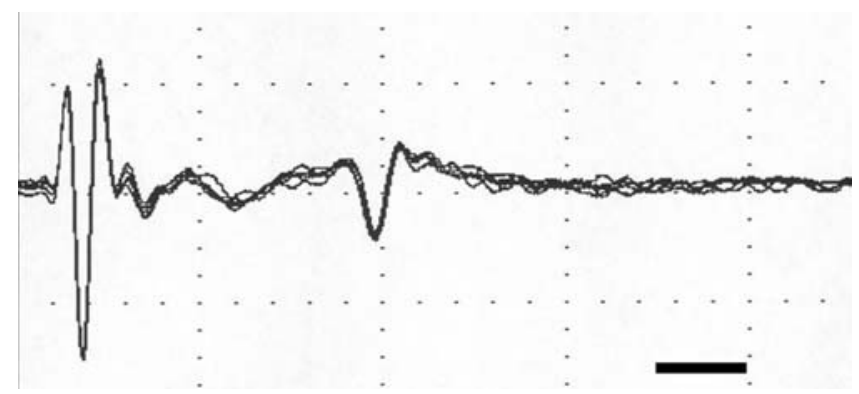

Fig. 4

Action potential evoked by electrical stimulation of the L5/L6 intervertebral disc. Black bar indicates $1 \mathrm{~ms}$.

variable location of the receptive fields. Only three were identified at the dorsal root ganglia, whose discharge pattern was sporadic and seemed not to be directly influenced by mechanical stimulation (Fig. 2). Consequently, we could not identify any units responding mechanically in the intervertebral disc at L5-L6. Meanwhile, spontaneous irregular bursting discharges, not responsive to any mechanical stimulation, were sometimes observed. These may be visceral sympathetic afferents (Fig. 3).

Responses to electrical stimuli. Because no distinct receptive field was detected in the discs and adjacent tissues, inter-vertebral discs were stimulated electrically to evoke the action potentials of the units. Overall, we identified 42 units with a mean conduction velocity of 7.86 (SD 4.9) m/s (2.56 to 21.6). Most of these velocities belonged to A-delta fibres with thin myelinated axons (Fig. 4).

Responses to inflammation. In the 19 rats subjected to carrageenan-induced inflammation of the L5-L6 intervertebral disc, the spontaneous baseline discharges increased slightly in five. Simultaneously, when the L5-L6 disc was stimulated mechanically, the responses were observed regardless of an absent response prior to the application of the inflammatory agent (Fig. 5).

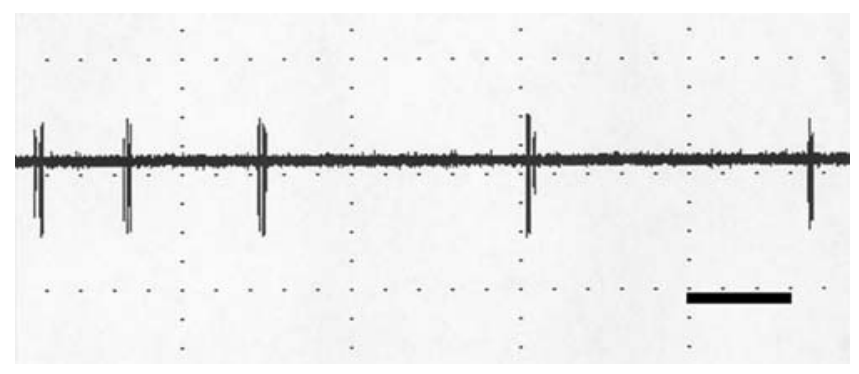

Fig. 3

Spontaneous irregular bursting discharge being insensitive to any mechanical stimulation. Black bar indicates $2 \mathrm{~s}$.

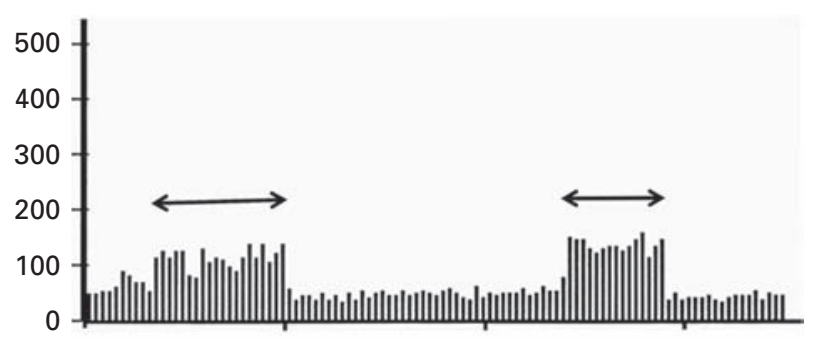

Fig. 5

Histogram showing multi-unit discharge rate changes in response to mechanical stimulation after inflammation. Black bar shows the interval from the onset to the end of the mechanical stimulation. Black bar indicates $5 \mathrm{~s}$ and arrow indicates mechanical stimulation.

\section{Discussion}

It has been reported that visceral and somatic primary afferent fibres had receptive fields in the low back region and projected stimuli centrally through the lumbar sympathetic trunk..$^{8,9,11,12}$ However, these studies have not demonstrated that the intervertebral discs themselves are the source of the low back pain. Using neurophysiological methods, this experiment has confirmed the presence of a sensory pathway of sympathetic afferent discharge from the dorsal aspect of the lower lumbar intervertebral discs to the dorsal roots of $\mathrm{L} 2$.

Conduction velocities were estimated from latencies evoked by bipolar electrical stimulation and the distance between the recording electrode and the point of electrical stimulation on the L5-L6 discs. This procedure lacks accuracy, possibly because the course of the afferent axons could not always be exactly determined. Nevertheless, we could not identify unmyelinated fibres with conduction velocities less than $2 \mathrm{~m} / \mathrm{s}$ possibly due to insufficient intensity of electric stimulation for evoking unmyelinated fibres.

In general, lumbar intervertebral discs have been considered as one of the major sources of low back pain. ${ }^{13,14}$ There is growing evidence that sympathetic afferents play a 
significant role in low back pain. Nakamura et a ${ }^{15}$ reported a study of 33 patients in whom infiltration of the L2 nerve roots with lidocaine relieved low back pain originating at the lower lumbar levels. On the basis of their previous neuroanatomical study, in which lumbar discs were found to have innervation from the sympathetic trunk, ${ }^{6}$ it was suggested that lumbar discogenic pain was a variety of visceral pain. Additionally, in the experimental setting, electrical stimulation of the lumbar sympathetic trunk has provoked low back pain ${ }^{16}$ and, conversely, lumbar sympathetic block reduced low back pain. ${ }^{17}$ The present study also showed that afferent signals originating from the L5-L6 intervertebral disc passed through the lumbar sympathetic trunk into the $\mathrm{L} 2$ roots and further into the spinal cord. These results suggest that lumbar discogenic pain is closely connected with the sympathetic afferent system.

Our experiment demonstrated that the L5-L6 intervertebral discs were not responsive to mechanical stimulation under normal conditions, but once inflamed by the application of carrageenan, some of these mechanically insensitive afferents did respond to mechanical stimulation. Mechanically insensitive afferents have been shown to become responsive to stimuli under pathological conditions such as inflammation in joints, ${ }^{18}$ viscera ${ }^{19}$ and the cornea. ${ }^{20}$ The receptors of mechanically insensitive afferent fibres are referred to as silent nociceptors and account for approximately half of the A-delta and $30 \%$ of the C-fibre nociceptors. ${ }^{21-24}$ These silent nociceptors have been described in the digestive system, related to the autonomic nerve fibres that partially pass through the sympathetic chain. The afferent fibres innervating the colon became sensitised to mechanical stimuli during inflammation. ${ }^{25}$ The findings of our study strongly suggest that the receptors of the intervertebral discs are silent nociceptors, which are activated under inflammatory conditions and modulate nociceptive information.

Kuslich, Ulstrom and Michael ${ }^{26}$ found that mechanical stimulation of the annulus of an affected disc during low back operations, conducted under local anaesthesia, provoked significant pain in approximately two-thirds of the symptomatic patients. However, the absence of pain in the remaining one-third remains unexplained. Similarly, discography conducted in a group of asymptomatic patients caused pain in only $10 \% .{ }^{27}$ To explain these clinical phenomena requires characterisation of the sensory information originating from the lower lumbar intervertebral discs, which is transmitted to the central nervous system. Recently, nerve growth has been found in granulation tissue in the fissures of degenerated discs. ${ }^{28}$ Our results may be a plausible explanation as to why mechanical stimulation to lumbar discs does not always produce pain. Inflammatory changes may cause the silent nociceptors to become responsive to mechanical stimuli, and this nociceptive information is transmitted as discogenic low back pain to the spinal cord through the sympathetic trunk.

It remains to be resolved how disc degeneration not associated with inflammation is correlated with low back pain.
Therefore, further analysis using a model of chronic disc degeneration is needed.

This study was supported by the NIH/NIAMS-AR41739 (JMC), the Orthopaedic and Education Foundation (CYC) and Wayne State University Office of Research.

No benefits in any form have been received or will be received from a commercial party related directly or indirectly to the subject of this article.

\section{References}

1. Bogduk N. The innervation of the lumbar spine. Spine 1983:8:286-93.

2. Groen GJ, Baljet B, Drukker J. Nerves and nerve plexuses of the human vertebral column. Am J Anat 1990;188:282-96.

3. Kojima Y, Maeda T, Arai R, Shichikawa K. Nerve supply to the posterior longitudinal ligament and the intervertebral disc of the rat vertebral column as studied by acetylcholinesterase histochemistry. I: distribution in the lumbar region. J Anat 1990; 169:237-46

4. Suseki K, Takahashi Y, Takahashi K, et al. Sensory nerve fibres from lumbar intervertebral discs pass through rami communicantes: a possible pathway for discogenic low back pain. J Bone Joint Surg [Br] 1998;80-B:737-4.

5. Morinaga T, Takahashi K, Yamagata M, et al. Sensory innervation to the anterior portion of lumbar intervertebral disc. Spine 1996;21:1848-51.

6. Nakamura S, Takahashi K, Takahashi Y, et al. Origin of nerves supplying the posterior portion of lumbar intervertebral discs in rats. Spine 1996;21:917-24.

7. Ohtori S, Takahashi Y, Takahashi K, et al. Sensory innervation of the dorsal portion of the lumbar intervertebral disc in rats. Spine 1999;24:2295-9.

8. Bahns E, Ernsberger U, Jänig W, Nelke A. Discharge properties of mechanosensitive afferents supplying the retroperitoneal space. Pflugers Arch 1986;407:519-25.

9. Cavanaugh JM, Kallakuri S, Chen CY, Ozaktäy AC. Sympathetic chain recordings from sensory nerves of the lumbar spine. Trans ORS 1997:692.

10. Pan HL, Longhurst JC. Ischemia-sensitive sympathetic afferents innervating the gastrointestinal tract function as nociceptors in cats. J Physio/ 1996;492:841-50

11. Gillette RG, Kramis RC, Roberts WJ. Sympathetic activation of cat spinal neurons responsive to noxious stimulation of deep tissues in the low back. Pain 1994;56:31-42.

12. Janig W, McLachlan EM. The sympathetic and sensory components of the caudal lumbar sympathetic trunk in the cat. J Comp Neurol 1986;245:62-73.

13. Mooney V. Where is the pain coming from? Spine 1987;12:754-9.

14. Nachemson AL. The lumbar spine: an orthopaedic challenge. Spine 1981;6:93-7.

15. Nakamura S, Takahashi K, Takahashi Y, Yamagata M, Moriya H. The afferent pathways of discogenic low back pain: evaluation of $\mathrm{L} 2$ spinal nerve infiltration. $J$ Bone Joint Surg [Br] 1996;78-B:606-12.

16. White JC, Sweet WH. Pain: its mechanisms and neurosurgical control. Springfield CC Thomas, 1955:67-98.

17. El Mahdi MA, Abdel Latif FY, Janko M. The spinal nerve root "innervation", and a new concept of the clinicopathological inter-relations in back pain and sciatica. Neurochirurgie 1981;24:137-41.

18. Schaible HG, Schmidt RF. Effects of an experimental arthritis on the sensory properties of fine articular afferent units. J Neurophysiol 1985;54:1109-22.

19. Habler H-J, Janig W, Koltzenburg M. A novel type of unmyelinated chemosensitive nociceptor in acutely inflamed urinary bladder. Agents Actions 1988;25:219-21.

20. Tanelian DL. Cholinergic activation of a population of corneal afferent nerves Exp Brain Res 1991:86:414-20.

21. Handworker HO, Kilo S, Reeh PW. Unresponsive afferent nerve fibers in the sural nerve of the rat. J Physiol 1991;435:229-42.

22. Kress M, Koltzenburg M, Reeh PW, Handwerker HO. Responsiveness and functional attributes of electrically localized terminals of cutaneous C-fibers in vivo and in vitro. J Neurophysiol 1992;68:581-95.

23. Meyer RA, Davis KD, Cohen RH, Treede RD, Campbell JN. Mechanically insensitive afferents (MIAs) in cutaneous nerves of monkey. Brain Res 1991;561:252-61.

24. Schmidt R, Schmelz M, Foster C, Ringkamp M, Torebjork HE. Novel classes of responsive and unresponsive C nociceptors in human skin. J Neurosci 1995;15:333-41.

25. Su X, Sengupta JN, Gebhart GF. Effects of kappa opioid receptor-selective agonists on responses of pelvic nerve afferents to noxious colorectal distension. J Neurophysiol 1997;78:1003-12.

26. Kuslich SD, Ulstrom CL, Michael CJ. The tissue origin of low back pain and sciatica: a report of pain response to tissue stimulation during operations on the lumbar spine using local anesthesia. Orthop Clin North Am 1991;22:181-7.

27. Carragee EJ, Tanner CM, Khurana S, et al. The rates of false-positive lumbar discography in select patients without low back symptoms. Spine 2000;11:1373-80

28. Peng B, Wu W, Hou S, et al. The pathogenesis of discogenic low back pain. J Bone Joint Surg [Br] 2005:87-B:62-7. 